\title{
Non Invasive Neuromodulation in Motor Recovery after Stroke: State of the Art, Open Questions and Future Perspectives
}

\author{
Raffaella Chieffo MD*, Giancarlo Comi MD and Letizia Leocani MD, PhD*
}

Neurological Department, Scientific Institute Vita-Salute University San Raffaele, Institute of Experimental Neurology, Milan, Italy

\begin{abstract}
Stroke is the leading cause of adult disability. Unfortunately, less than $40 \%$ of stroke survivors completely recover, despite intensive acute care and rehabilitation training. Non invasive brain stimulation (NIBS) techniques have been recognized as a promising intervention to improve motor recovery after stroke. Repeated sessions of repetitive transcranial magnetic stimulation (rTMS) and transcranial direct current stimulation (tDCS) can, indeed, induce changes in cortical excitability and long term plasticity. Several protocols of stimulation have been already tested and proven efficient in modulating the lesioned as well as the unlesioned hemisphere after stroke. However, not all patients can be considered as responder to NIBS. We provide an overview of the rationale, open questions and future perspectives for NIBS after stroke.
\end{abstract}

Keywords: Neuromodulation; Stroke: Rehabilitation; Motor recovery

\section{Introduction}

Stroke is a leading cause of adult disability in the western world [1]. Sensorimotor and cognitive impairments often have a great impact on quality of life in post stroke survivors. It is well known that the human brain continues to adjust throughout life. After ischemic brain injury, neuroplasticity is particularly active in the first months [2]. However, it has been demonstrated that, even years after stroke, the human brain still retains the capacity to reorganize in response to interventions influencing motor recovery [3]. Several efforts have focused on the development of new restorative therapies. In particular, non invasive brain stimulation (NIBS) techniques, such as transcranial magnetic stimulation (TMS) and transcranial direct current stimulation (tDCS), constitute an extensive chapter on this topic. The rationale for the application of NIBS for rehabilitation of neurological deficits is its capacity of modulate cortical excitability and induce plasticity in humans. Repetitive TMS (rTMS) refers to regularly repeated single TMS pulses delivered in trains at specific frequencies. In general, low-frequency rTMS $(\leq 1 \mathrm{~Hz})$ usually results in cortical excitability reduction [4] in healthy subjects, whereas with higher frequencies (5 $\mathrm{Hz}$ or more) cortical excitability is usually increased [5]. Modulation by rTMS does not only depend on pulses frequency. Intensity of stimulation, trains duration and inter-train wait time can be all manipulated to influence neuroplasticity [6]. Theta-burst stimulation (TBS) is a relative more recent technique, consisting in brief bursts of theta frequency low intensity stimuli. TBS delivered in a continuous pattern (cTBS) produces a decrease in motor cortex excitability, while an intermitted paradigm (iTBS) produces an opposite effect [7]. The so called tDCS consists in the application of weak electrical currents through the scalp. This technique utilizes two surface electrodes (anode and cathode), which placement is fundamental for outcome, by determining the direction of the current flow. The montage with anode on the brain region of interest (anodal tDCS) has an excitatory effect (and cathodal tDCS the opposite), possibly through mechanisms involving neuronal membrane depolarization or hyperpolarization respectively $[8,9]$.

In general, a single session of NIBS induces reversible effects that last from a few minutes up to about $1 \mathrm{~h}$. To induce long lasting effects several stimulation sessions are often needed. The mechanisms underlying long-term effects of rTMS are not completely understood.
It is likely that a number of interacting mechanisms are involved. There are several evidences that the effects of these NBS techniques are mainly due to long term potentiation (LTP)-like and long term depression (LTD)-like mechanisms [10-13]. Moreover, experiments performed in healthy rat brain shown that NIBS has the ability to mediate neural plasticity by enhancing the expressions of neurotransmitters and neurotrophins, such as glutamate, N-methyl-D-aspartate (NMDA), and brain-derived neurotrophic factor (BDNF) [14-16].

Considering safety, because of the facilitatory effect of highfrequency rTMS, the major concern with its use is the risk of inducing seizures. Luckily, this is a quite rare adverse effect (risk estimate of $1.4 \%$ in epileptic patients, less than $1 \%$ in healthy subjects) [17]. Seizures can be induced by rTMS when pulses are applied with relatively high frequencies and short interval periods between trains of stimulation that should be always set at more than 1 second [18]. For tDCS, the main problem reported is transient skin reactions below the stimulating electrodes. To effectively minimize risks, safety guidelines have been issued regarding stimulation parameters for both rTMS and tDCS $[17,19,20]$.

\section{NIBS and Upper Limb Motor Recovery}

Positron emission tomography (PET) and functional magnetic resonance imaging (fMRI) studies show increase activation of homologous motor areas and secondary motor regions involving both hemispheres during movement of the paretic hand in the acute and postacute phases after stroke [21-23]. The degree of such motor overflow correlated with severity of motor deficit as well as with poor motor outcome suggesting a maladaptive plastic reorganization of the

*Corresponding author: Letizia Leocani MD, PhD and Raffaella Chieffo MD Neurological Department and Institute of Experimental Neurology - INSPE, Scientific Institute Hospital San Raffaele, Milan, Italy, Tel: +39-02-2643-3092; Fax: +39-02-2643-3085; E-mail: letizia.leocani@hsr.it

Received September 03, 2013; Accepted October 17, 2013; Published October 25,2013

Citation: Chieffo R, Comi G, Leocani L (2013) Non Invasive Neuromodulation in Motor Recovery after Stroke: State of the Art, Open Questions and Future Perspectives. J Neurol Neurophysiol 4: 168. doi:10.4172/2155-9562.1000168

Copyright: ( 2013 Chieffo R, et al. This is an open-access article distributed under the terms of the Creative Commons Attribution License, which permits unrestricted use, distribution, and reproduction in any medium, provided the original author and source are credited. 
unaffected hemisphere after stroke [24]. Also in neurophysiological studies an early disinhibition of the motor pathways has been demonstrated. Interhemispheric inhibitory circuits resulted, indeed, altered after monohemispheric stroke $[25,26]$ as well as intracortical inhibition (ICI) over affected and unaffected hemisphere was found reduced in stroke studies [27-30]. Mapping the motor cortex with TMS offers the possibility to study acute and rapid plastic rearrangements of cortical motor output in physiological or pathological conditions [31]. An enlargement of the map of output from the unlesioned primary motor cortex to the contralateral hand has been documented in the subacute phases of cortical and subcortical stroke $[32,33]$.

Although acute hyperexcitability of the unaffected hemisphere tends to recover over time, it has a negative prognostic value on motor recovery and it has been associated with a transitory motor impairment of the non paretic hand [32]. Overall, these observations suggest a diffuse and bilateral distress of the motor system occurring after acute stroke. As described by Ward and Cohen (2004) and more recently stated by Nowak et al (2009), the hypothesis of interhemispheric competition proposes that post stroke motor deficits are due to reduced output from the damaged hemisphere not only because of infarct itself but also because of excessive transcallosal inhibition from the intact hemisphere. Motor performance also depends on competitive inhibitory processes between both hemispheres [22,34]. According to the concept of interhemispheric competition, NIBS have been mostly finalized in acute as well as in chronic stroke patients to induced inhibition of M1 of the contralesional hemisphere (low frequency rTMS, cTBS, cathode tDCS) or facilitation of excitability of M1 of the ipsilesional hemisphere (hight frequency rTMS, iTBS, anodal tDCS) in order to normalize the interhemispheric balance through transcallosal connections and to promote output from the lesioned motor cortex [35]. It is necessary to specify that, in the chronic phases after stroke, interhemispheric competition would be less pronounced than in the earlier subacute period, as suggested by the finding that both transcallosal asymmetry [36] and hyperxcitability of the unlesioned hemisphere [32] slow down with time. Although the optimal recovery after stroke well correlates with the reacquisition of innervations from areas surrounding the lesion, in contrast to activation at more distant locations [24], it is possible that the reorganization of the non primary regions in the post-acute or chronic phases may play a constructive role via an interaction with surviving corticospinal tract from the affected hemisphere $(\mathrm{AH})$. Consistently with this view, disruption of activity in contralesional M1 did not greatly impair hand function in patients with chronic subcortical stroke and good motor recovery [37]. For example temporary interference of ipsilesional dorsolateral premotor cortex (PMd) [38] and even more of contralesional PMd [39] using TMS appears to worsen performance of a simple hand motor task in chronic subcortical stroke patients but not in healthy controls.

\section{Inhibition of the Unlesioned Hemisphere}

The down-regulation of the unlesioned M1 seems to be effective in the subacute phases and in the chronic phases post stroke as well. Nowak and colleagues (2008) evaluated the effect on fMRI neural activation of a single session of $1 \mathrm{~Hz}$ rTMS over the unlesioned M1 in fifteen patients affected by subcortical stroke. They found that rTMS applied to the contralesional M1 improves the kinematics of finger and grasp movements in the affected hand and reduces the overactivity in the contralesional primary and non primary motor areas during a single motor task performed with the paretic hand [40]. Changes in cortical activity are likely related to modifications of intracortical excitabily and interhemispheric connections. Indeed, it has been demonstrated that low frequency rTMS reduces the amplitude of motor evoked potentials (MEPs) in contralesional M1, increases excitability in the affected hemisphere and reduces transcallosal inhibition (TCI) from the unaffected toward the affected hemisphere [41,42]. Moreover, the improvement in motor function after $1 \mathrm{~Hz}$ rTMS seems to significantly correlate with a reduced TCI duration. The estimated effect size of a single session of low-frequency rTMS or cathodal tDCS on upper limb motor function ranges from $10 \%$ to $60 \%$ improvement [43]. This effect, in line with effects on cortical motor excitability in human [6,44-46] and animal models [47], is however transitory and outlasts the stimulation period from minutes to $1-2$ hours. Multiple stimulation sessions and/ or the association of a motor training are needed to induce longer lasting effects on cortical excitability and motor function (2-4 weeks) [42,48-50]. In particular, Avenanti et al. (2012) evaluated the effect of 10 daily sessions of $1 \mathrm{~Hz}$ rTMS administered either immediately before or after physiotherapy (PT), in 30 subjects affected by chronic subcortical stroke. Treatment induced cumulative rebalance of excitability between the two hemispheres and a reduction of interhemispheric inhibition in comparison PT alone. Moreover, greater and more stable behavioural and neurophysiologic outcomes were found in the group of patients receiving rTMS before PT, whereas the application of rTMS after PT showed a slight improvement that declined over time [51]. On the contrary, cTBS of the unlesioned hemisphere seems to be not effective. Tadelli et al. in 2007 found that cTBS suppressed the MEPs evoked in the healthy hands but did not change motor behaviour or the electrophysiology of the paretic hands in 6 chronic stroke patients [52]. In another study (10 chronic subcortical stroke) paretic upper limb motor function was even impaired by cTBS of the contralesional $\mathrm{M} 1$, and this was correlated with reduced ipsilesional corticomotor excitability [53].

\section{Facilitation of the Lesioned Hemisphere}

The facilitation of the affected hemisphere appears to be a useful approach to enhance motor function of the paretic limb after stroke and several NIBS protocols have been tested $(3 \mathrm{~Hz}, 5 \mathrm{~Hz}, 10 \mathrm{~Hz}, 20 \mathrm{~Hz}$ rTMS, iTBS, anodal tDCS). Increased of motor cortical excitability within the affected hemisphere, expressed as MEPs amplitude or recruitment curves increase and reduced short interval intracortical inhibition, has been observed after iTBS, anothal tDCS or high frequency rTMS $[52,54,55]$. The estimated effect size to improve paretic motor function ranges from $10 \%$ to $150 \%$ for all these methods [43]. Besides differences in the techniques and in the outcome measurements used in these studies, the large variability in results could be influenced by other factors. Amelie and collegues (2009) compared the effect of $10 \mathrm{~Hz}$ rTMS over ipsilesional M1 in cortical and subcortical stroke patients. Interestingly, they found that $10 \mathrm{~Hz}$ rTMS over the ipsilesional M1, but not over the vertex, improved movement kinematics in 14 of 16 patients with subcortical stroke [56]. On the contrary, rTMS slightly deteriorated dexterity of the affected hand in 7 of 13 cortical stroke patients. These two behavioural effects were associated with different fMRI neural activation patterns. In subcortical stroke, rTMS over ipsilesional M1 reduced neural activity of the contralesional M1, while in cortical stroke caused a widespread bilateral recruitment of primary and secondary motor areas [56]. Moreover, activity in ipsilesional M1 at baseline correlated with rTMS-induced improvement of finger tapping frequency, suggesting that the effectiveness of facilitatory rTMS applied over ipsilesional M1 depends on the functional integrity of the stimulation site and/or the extent of the brain area affected by the stroke [56]. Accordingly, a significant improvement of the paretic upper limb motor function was observed after subcortical stroke, by applying anodal tDCS over the M1 of the affected hemisphere in contrast with little effects in the presence of cortical lesions [57]. A 
recent meta-analysis showed that NIBS techniques have similar effect in improving recovery in the acute $(<2$ weeks, but often $>1$ week), subacute ( 2 weeks to 6 months) and chronic (> 6 months) phases after stroke [58]. However, a double blind, sham-controlled study on 25 patients after acute stroke undergoing anodal tDCS stimulation (vs 25 undergoing sham) on the affected hemisphere from the second day after acute stroke for five days, failed to demonstrate a significant advantage compared with the sham stimulation group on NIHSS and Fugl-Meyer motor scale [59].

As observed with inhibitory stimulation of the unlesioned hemisphere, also the facilitation of the affected hemisphere trough NIBS seems to be a therapeutic adjuvant to motor training techniques such as conventional physical and occupational therapy, reaching and grasping exercises, robot-assisted arm training [57,60-62]. On the contrary, no significant synergic effects of $20 \mathrm{~Hz}$ rTMS over the M1 of the lesioned hemisphere combined with constraint-induced therapy (CIMT) were found in comparison with CIMT alone [63]. Clinical effects of high-frequency rTMS in 17 patients with hemiparesis after stroke (onset $>5$ months) have been investigated together with neural correlates [64]. Ten daily sessions of 1000 pulses of real or sham rTMS were applied at $10 \mathrm{~Hz}$ over the primary motor cortex of the affected hemisphere, each fifty-pulse train was followed by sequential finger motor training of the paretic hand. Movement accuracy of sequential motor tasks showed greater improvement in the real group than in the sham group, whereas fMRI acquisitions highlighted how patients in the real rTMS group significantly enhanced activation in the affected hemisphere compared to the sham rTMS group [64]. According to these results, high-frequency rTMS coupled with motor training improved motor performance through modulation of activities in the cortico-basal ganglia-thalamocortical circuits.

\section{Bilateral Stimulation Protocol}

Both anodal tDCS over the ipsilesional M1 and cathodal tDCS stimulation of the contralesional M1 have been shown to induce amelioration of motor performance of the paretic upper limb as well as changes in cortical excitability [35]. More recently, it has been hypothesized that an additive effect could be produced delivering anodal and cathodal tDCS simultaneously [65]. Positioning the anode over one motor cortex and the cathode over the contralateral motor cortex can induce an increase in cortical excitability on the anodal a decrease in the cathodal stimulated side [66]. Two sham-controlled studies showed a significant improvement of the paretic upper limb motor function in chronic stroke after 1 and 5 consecutive sessions of bihemispheric tDCS (anodal tDCS of the ipsilesional M1 and cathodal tDCS of the contralesional M1) combined with physical therapy $[67,68]$. A stronger fMRI activation of intact ipsilesional motor regions during movements of the affected limb has been found after 5 session of real treatment whereas no significant activation changes were seen in the control group [67]. These studies suggest that the bihemispheric tDCS application combined with physical therapy may be an ideal strategy to generate functional improvement in stroke patients. However, comparing the effect of the 3 different tDCS montages, more recently findings indicate the superiority of anodal tDCS or cathodal tDCS over bilater $\mathrm{tDCS}$ in improving upper limb motor function in chronic stroke patients $[69,70]$. Also in healthy subjects, anodal and cathodal tDCS induce greater changes in cortical excitability in comparison with bilateral tDCS $[66,70]$.

\section{Open Questions}

Recent studies performed on a larger number of subjects failed to demonstrate the efficacy of NIBS techniques in post stroke motor recovery of the paretic upper limb $[59,71,72]$. These findings are indicative of the fact that not all the subjects may benefit from the application of the different NIBS techniques. Could be, therefore, useful to select patients best responder to NIBS. The identification of clinical, functional, neurophysiological and neurochemical markers of behavioural response to NIBS should help to predict patient outcome. As previously discussed stroke features are fundamental to predict response to NIBS. Effectiveness of facilitatory stimulation of the ipsilesional M1 depends on the functional integrity of the stimulation site and/or the extent of the brain area affected by stroke [56]. In general, a greater effectiveness of NIBS in subcortical stroke patients in comparison with patients with non specified lesion site has been confirmed in a recent meta-analysis [58]. Also baseline severity of the affected upper limb significantly influenced the treatment outcome, greatest results have been observed for less impaired patients [49]. The neurochemical predictors of behavioural response to tDCS after stroke have been searched using magnetic resonance spectroscopy [70]. Higher GABA levels in the ipsilesional M1 - and not in the occipital region - predicted subsequent behavioural gains by anodal tDCS, as measured using reaction time [70]. The authors argued that, as in healthy controls anodal tDCS induces a local reduction in M1 GABA levels [73], patients with high M1 GABA levels may have a higher potential dynamic range for GABA modification by Anodal tDCS [70]. The same authors did not find evidence of correlation between basal M1 GABA and its modification by anodal tDCS in health volunteers [74]. However, even in healthy subjects there is high variability in the neurophysiological and behavioural response to brain stimulation techniques. Many of the factors involved are still unknown, the identified factors have been, instead, summarized in a review by Ridding and Ziemann. Besides some non modifiable factors such as gender, age and genetic (BDNF polymorphism Val66Met), others can be manipulated such as time of the day of the stimulation session, concurrent aerobic exercise, pharmacological intake, attention. More recently, Humada and colleagues hypothesized that different responses to rTMS should be related to individual differences in recruitment of cortical neurons. They examined the effect of inhibitory and excitatory TBS in 56 healthy subject and they found that the effect of TBS was highly correlated with the efficiency of late I-wave recruitment. These results indicate that variation in response to rTMS is influenced by which interneuron networks are recluted by TMS pulse [75]. Further studies are needed to better understand the physiological mechanisms conditioning the response to NIBS techniques.

\section{NIBS and Lower Limb Motor Recovery}

Although the ability to walk is impaired in more than $80 \%$ of poststroke patients [76], more efforts have focused on the recovery of the paretic upper extremity than on the recovery of lower limb function. This might be mainly due to functional and anatomical limitations. Concerning the latter, lower limb cortical motor areas are located close to the midline into the depth of the medial longitudinal fissure. These areas are less easily approached with NIBS techniques, especially when dealing with distal muscles. Pathophysiological reorganization of leg motor areas after stroke is still unclear. A study performed with near-infrared spectroscopic imaging system (NIRS) in stroke patients during walking showed that, similarly to upper limb, the cortical activation patterns of motor, premotor and supplementary lower limb motor cortex was greater for the unaffected rather than for the affected hemisphere [77]. Improvements of gait parameters of the paretic lower limb have been found associated with a reduction of 
the interhemispheric asymmetry of the primary sensorimotor cortical activations [78].

\section{Inhibition of the Unlesioned Hemisphere}

Based on the interhemispheric asymmetry between lesioned and unlesioned hemisphere observed after stroke, Jayaram and Stinear evaluated the effect of different NIBS protocols applied during walking on the lower limb motor cortex excitability. They tested the inhibitory paired associative stimulation (iPAS) and the inhibitory $1 \mathrm{~Hz}$ rTMS applied to preferentially stimulate the unaffected lower limb, as well as the excitatory anodic tDCS applied with electrodes offset to preferentially stimulate the affected M1. They found that all NIBS protocols were effective in modulating excitability of both lower limb motor areas. The modulator effects consisted in the increase of MEPs amplitude over the paretic limb and in the decrease of MEP amplitude over the non paretic limb [79]. No behavioural effects were tested in this study. Wang and colleagues first evaluated, in chronic stroke patients, the clinical effect of task-oriented training associated with $1 \mathrm{~Hz}$ repetitive transcranial magnetic stimulation (rTMS) performed to inhibit the unaffected lower limb motor cortex. The authors performed a sham controlled study, using a figure-of-eight coil tangentially positioned over the leg area at the optimal site for response from the rectus femoris muscle. They showed that 10 daily sessions of rTMS enhanced the effect of task-oriented training on walking performance and motor control ability thereby leading to a more symmetric gait pattern. Moreover, rTMS improved the symmetry of interhemispheric corticomotor excitability [80].

\section{Facilitation of the Lesioned Hemisphere}

The effect of facilitatory tDCS on fine motor control of the paretic foot was evaluated by applying anodal or sham tDCS in a random order over the lower limb primary motor cortex of the lesioned or non-lesioned hemisphere. In each session, tDCS was associated with a motor task consisting in tracking a sinusoidal waveform for 15 min using dorsiflexion-plantarflexion movements of their paretic ankle. Anodal tDCS over the affected hemisphere enhanced the task practice effect already revealed in the sham stimulation condition. tDCS applied over the unlesioned hemisphere eliminated, instead, the task-induced practice effect [81]. The facilitation of the unlesioned hemisphere alone seems to be, therefore, detrimental for the paretic lower limb performance. On the contrary, the simultaneous facilitation of both lower limb motor areas, performed with high frequency rTMS connected to a double cone coil, has been reported to significantly improve walking in chronic gait involvement following stroke [82,83]. To date, few studies evaluated the interhemispheric connections between the two lower limb motor areas. However, a bi-hemispheric control of foot movements have been hypothesized in healthy subjects [84]. During voluntary movement, a stronger lateralization of fMRI activation has been reported for finger movements in comparison with lower limb joints [85]. The latter finding suggests a different functional specialization and organization between brain circuits controlling hand and foot movement. Therefore, the hypothesis of inter-hemispheric competition in unilateral motor control, supported by converging evidence from studies on the upper limb, may not be transposed directly to the lower limb motor system.

\section{Development of New Devices for NIBS}

New devices for both rTMS and tDCS have been developed in the last years and may be further explored for their potential application in stroke research. In 2002, a new TMS coil, termed Hesed coil (H-coil), has been introduced [82] to effectively stimulate deep brain regions without increasing the electric field intensity in the superficial cortical regions [86]. The basic concept is to obtain summation of the electric field in depth, minimizing the current components that only cause accumulation of surface charge. Moreover, the drop of the induced field as a function of the distance is much slower compared to a double-cone coil. The H-coil efficacy in humans was tested by Zangen and colleagues on six healthy volunteers [83]. They evaluated the rate of decrease of the electric field by gradually increasing the coil distance from the skull and measuring the abductor pollicis brevis (APB) motor threshold at each distance. The motor cortex could be activated by a specific version of the $\mathrm{H}$-coil at a distance of $5.5 \mathrm{~cm}$ vs $2 \mathrm{~cm}$ with the figure-of-eight coil [87]. Safety of H-coils has also been assessed by Levkovitz et al. at relatively high intensity ( $120 \%$ of motor threshold) [84]. Thirty-two healthy volunteers were tested in a pre-post design during three (single pulses, $10 \mathrm{~Hz}$, and $20 \mathrm{~Hz}$ ) stimulation sessions made with two $\mathrm{H}$-coil designs $(\mathrm{H} 1 / \mathrm{H} 2)$, standard figure-of- 8 coil, and sham-coil control. $\mathrm{H}$-coils were well tolerated, with no adverse physical or neurological outcomes [88]. The H-coils designed have been preferentially tested to evaluate the efficacy in cognitive and mood alteration in psychiatric and neurological diseases [89-91]. Using a $\mathrm{H}$-coil designed to target the lower limb motor cortex, analgesic effects have been obtained in patients with painful diabetic neuropathy [92]. Contrary to what observed with TMS, the standard tDCS electrode configurations deliver a wider and superficial electric filed. The so called high-definition (HD)-tDCS is a new approach finalized to improve spatial focality and to optimize intensity of stimulation at target $[93,94]$. It consists in 5 electrodes of smaller size displaced in a concentric-ring configuration with the polarity (anode or cathode) set by a centre electrode and the area of cortical modulation restricted by adjusting the radii of 4 return electrodes [93,94]. In particular, high-resolution MRI-based forward models have been used to probe the unidirectional and targeted stimulation of the $4 \mathrm{x} 1$-ring montage of HD-tDCS. Although data on the application of these techniques to improve stroke outcome are not available, further research may explore these new directions.

\section{Acknowledgments}

Partially supported by the Joint Italian-Israeli laboratory San RaffaeleWeizmann (Italian Ministry of Foreign Affairs). Dr Chieffo participated in this work as partial fulfilment of her PhD in Molecular Medicine, Program in Experimenta Neurology, San Raffaele University, Milan, Italy.

\section{References}

1. Roger VL, Go AS, Lloyd-Jones DM, Benjamin EJ, Berry JD, et al. (2012) Heart disease and stroke statistics -2012 update: a report from the American Heart Association. Circulation 125: e2-e220.

2. Nudo RJ (2003) Functional and structural plasticity in motor cortex: implications for stroke recovery. Phys Med Rehabil Clin N Am 14: S57-76.

3. Liepert J, Miltner WH, Bauder H, Sommer M, Dettmers C, et al. (1998) Motor cortex plasticity during constraint-induced movement therapy in stroke patients. Neurosci Lett 250: 5-8.

4. Chen R, Classen J, Gerloff C, Celnik P, Wassermann EM, et al. (1997) Depression of motor cortex excitability by low-frequency transcranial magnetic stimulation. Neurology 48: 1398-1403.

5. Pascual-Leone A, Valls-Solé J, Wassermann EM, Hallett M (1994) Responses to rapid-rate transcranial magnetic stimulation of the human motor cortex. Brain $117: 847-858$

6. Hallett M (2000) Transcranial magnetic stimulation and the human brain Nature 406: 147-150.

7. Huang YZ, Edwards MJ, Rounis E, Bhatia KP, Rothwell JC (2005) Theta burst stimulation of the human motor cortex. Neuron 45: 201-206 .

8. Priori A, Berardelli A, Rona S, Accornero N, Manfredi M (1998) Polarization of the human motor cortex through the scalp. Neuroreport 9: 2257-2260. 
9. Nitsche MA, Paulus W (2000) Excitability changes induced in the human moto cortex by weak transcranial direct current stimulation. J Physiol 527 Pt 3: 633639.

10. Cooke SF, Bliss TV (2006) Plasticity in the human central nervous system. Brain 129: 1659-1673.

11. Thickbroom GW (2007) Transcranial magnetic stimulation and synaptic plasticity: experimental framework and human models. Exp Brain Res 180: 583-593.

12. Wagner T, Valero-Cabre A, Pascual-Leone A (2007) Noninvasive human brain stimulation. Annu Rev Biomed Eng 9: 527-565.

13. Ziemann U, Paulus W, Nitsche MA, Pascual-Leone A, Byblow WD, et al. (2008) Consensus: Motor cortex plasticity protocols. Brain Stimul 1: 164-182.

14. Kole MH, Fuchs E, Ziemann U, Paulus W, Ebert U (1999) Changes in 5-HT1A and NMDA binding sites by a single rapid transcranial magnetic stimulation procedure in rats. Brain Res 826: 309-312.

15. Muller MB, Toschi N, Kresse AE, Post A, Keck ME (2000) Long-term repetitive transcranial magnetic stimulation increases the expression of brain-derived neurotrophic factor and cholecystokinin mRNA, but not neuropeptide tyrosine mRNA in specific areas of rat brain. Neuropsychopharmacology 23: 205-215.

16. Gersner R, Kravetz E, Feil J, Pell G, Zangen A (2011) Long-term effects of repetitive transcranial magnetic stimulation on markers for neuroplasticity: differential outcomes in anesthetized and awake animals. J Neurosci 31: 7521

17. Rossi S, Hallett M, Rossini PM, Pascual-Leone A; Safety of TMS Consensus Group (2009) Safety, ethical considerations, and application guidelines for the use of transcranial magnetic stimulation in clinical practice and research. Clin Neurophysiol 120: 2008-2039.

18. Chen R, Gerloff C, Classen J, Wassermann EM, Hallett M, et al. (1997) Safety of different inter-train intervals for repetitive transcranial magnetic stimulation and recommendations for safe ranges of stimulation parameters. Electroencephalogr Clin Neurophysiol 105: 415-421.

19. Nitsche MA, Cohen LG, Wassermann EM, Priori A, Lang N, et al. (2008) Transcranial direct current stimulation: State of the art 2008. Brain Stimul 1: 206-223

20. Priori A, Hallett M, Rothwell JC (2009) Repetitive transcranial magnetic stimulation or transcranial direct current stimulation? Brain Stimul 2: 241-245.

21. Chollet F, DiPiero V, Wise RJ, Brooks DJ, Dolan RJ, et al. (1991) The functional anatomy of motor recovery after stroke in humans: a study with positron emission tomography. Ann Neurol 29: 63-71.

22. Ward NS, Cohen LG (2004) Mechanisms underlying recovery of motor function after stroke. Arch Neurol 61: 1844-1848.

23. Calautti C, Naccarato M, Jones PS, Sharma N, Day DD, et al. (2007) The relationship between motor deficit and hemisphere activation balance after stroke: A 3T fMRI study. Neuroimage 34: 322-331.

24. Ward NS, Brown MM, Thompson AJ, Frackowiak RS (2003) Neural correlates of outcome after stroke: a cross-sectional fMRI study. Brain 126: 1430-1448.

25. Ferbert A, Priori A, Rothwell JC, Day BL, Colebatch JG, et al. (1992) Interhemispheric inhibition of the human motor cortex. J Physiol 453: 525-546.

26. Bütefisch CM, Wessling M, Netz J, Seitz RJ, Hömberg V (2008) Relationship between interhemispheric inhibition and motor cortex excitability in subacute stroke patients. Neurorehabil Neural Repair 22: 4-21.

27. Shimizu T, Hosaki A, Hino T, Sato M, Komori T, et al. (2002) Motor cortical disinhibition in the unaffected hemisphere after unilateral cortical stroke. Brain 125: 1896-1907.

28. Manganotti P, Acler M, Zanette GP, Smania N, Fiaschi A (2008) Motor cortical disinhibition during early and late recovery after stroke. Neurorehabil Neural Repair 22: 396-403.

29. Liepert J, Hamzei F, Weiller C (2000) Motor cortex disinhibition of the unaffected hemisphere after acute stroke. Muscle Nerve 23: 1761-1763.

30. Bütefisch CM, Netz J, Wessling M, Seitz RJ, Hömberg V (2003) Remote changes in cortical excitability after stroke. Brain 126: 470-481.

31. Traversa R, Cicinelli P, Bassi A, Rossini PM, Bernardi G (1997) Mapping of motor cortical reorganization after stroke. A brain stimulation study with focal magnetic pulses. Stroke 28: 110-117.
32. Chieffo R, Inuggi A Straffi L, Coppi E, Gonzalez-Rosa J, et al. (2013) Mapping early changes of cortical motor output after subcortical stroke: a transcranial magnetic stimulation study. Brain Stimul 6: 322-329.

33. Delvaux V, Alagona G, Gérard P, De Pasqua V, Pennisi G, et al. (2003) Poststroke reorganization of hand motor area: a 1-year prospective follow-up with focal transcranial magnetic stimulation. Clin Neurophysiol 114: 1217-1225.

34. Nowak DA, Grefkes C, Ameli M, Fink GR (2009) Interhemispheric competition after stroke: brain stimulation to enhance recovery of function of the affected hand. Neurorehabil Neural Repair 23: 641-656.

35. Hummel FC, Cohen LG (2006) Non-invasive brain stimulation: a new strategy to improve neurorehabilitation after stroke? Lancet Neurol 5: 708-712.

36. Swayne OB, Rothwell JC, Ward NS, Greenwood RJ (2008) Stages of motor output reorganization after hemispheric stroke suggested by longitudinal studies of cortical physiology. Cereb Cortex 18: 1909-1922.

37. Werhahn KJ, Conforto AB, Kadom N, Hallett M, Cohen LG (2003) Contribution of the ipsilateral motor cortex to recovery after chronic stroke. Ann Neurol 54 464-472.

38. Fridman EA, Hanakawa T, Chung M, Hummel F, Leiguarda RC, et al. (2004) Reorganization of the human ipsilesional premotor cortex after stroke. Brain 127: $747-758$.

39. Johansen-Berg H, Rushworth MF, Bogdanovic MD, Kischka U, Wimalaratna S, et al. (2002) The role of ipsilateral premotor cortex in hand movement afte stroke. Proc Natl Acad Sci U S A 99: 14518-14523.

40. Nowak DA, Grefkes C, Dafotakis M, Eickhoff S, Küst J, et al. (2008) Effects of low-frequency repetitive transcranial magnetic stimulation of the contralesional primary motor cortex on movement kinematics and neural activity in subcortical stroke. Arch Neurology 65: 741-747.

41. Takeuchi N, Chuma T, Matsuo Y, Watanabe I, Ikoma K (2005) Repetitive transcranial magnetic stimulation of contralesional primary motor cortex improves hand function after stroke. Stroke 36: 2681-2686.

42. Fregni F, Boggio PS, Valle AC, Rocha RR, Duarte J, et al. (2006) A shamcontrolled trial of a 5-day course of repetitive transcranial magnetic stimulation of the unaffected hemisphere in stroke patients. Stroke 37: 2115-2122.

43. Nowak DA, Bösl K, Podubeckà J, Carey JR (2010) Noninvasive brain stimulation and motor recovery after stroke. Restor Neurol Neurosci 28: 531-544.

44. Paulus W (2003) Transcranial direct current stimulation (tDCS). Suppl Clin Neurophysiol 56: 249-254.

45. Nitsche MA, Liebetanz D, Antal A, Lang N, Tergau F, et al. (2003) Modulation of cortical excitability by weak direct current stimulation--technical, safety and functional aspects. Suppl Clin Neurophysiol 56: 255-276.

46. Siebner HR, Rothwell J (2003) Transcranial magnetic stimulation: new insights into representational cortical plasticity. Exp Brain Res 148: 1-16.

47. Cambiaghi M, Velikova S, Gonzalez-Rosa JJ, Cursi M, Comi G, et al. (2010) Brain transcranial direct current stimulation modulates motor excitability in mice. Eur J Neurosci 31: 704-709.

48. Boggio PS, Nunes A, Rigonatti SP, Nitsche MA, Pascual-Leone A, et al. (2007) Repeated sessions of noninvasive brain DC stimulation is associated with motor function improvement in stroke patients. Restor Neurol Neurosci 25 123-129.

49. Kakuda W, Abo M, Shimizu M, Sasanuma J, Okamoto T, et al. (2012) A multicenter study on low-frequency rTMS combined with intensive occupational therapy for upper limb hemiparesis in post-stroke patients. J Neuroeng Rehabi 9: 4

50. Seniow J, Bilik M, Lesniak M, Waldowski K, Iwanski S, et al. (2012) Transcranial magnetic stimulation combined with physiotherapy in rehabilitation of poststroke hemiparesis: a randomized, double-blind, placebo-controlled study. Neurorehabil Neural Repair 26: 1072-1079.

51. Avenanti A, Coccia M, Ladavas E, Provinciali L, Ceravolo MG (2012) Lowfrequency rTMS promotes use-dependent motor plasticity in chronic stroke: a randomized trial. Neurology 78: 256-264.

52. Talelli P, Greenwood RJ, Rothwell JC (2007) Exploring Theta Burst Stimulation as an intervention to improve motor recovery in chronic stroke. Clin Neurophysio 118: 333-342.

53. Ackerley SJ, Stinear CM, Barber PA, Byblow WD (2010) Combining theta burs stimulation with training after subcortical stroke. Stroke 41: 1568-1572. 
Citation: Chieffo R, Comi G, Leocani L (2013) Non Invasive Neuromodulation in Motor Recovery after Stroke: State of the Art, Open Questions and Future Perspectives. J Neurol Neurophysiol 4: 168. doi:10.4172/2155-9562.1000168

54. Hummel F, Celnik P, Giraux P, Floel A, Wu WH, et al. (2005) Effects of noninvasive cortical stimulation on skilled motor function in chronic stroke. Brain 128: 490-499.

55. Kim YH, You SH, Ko MH, Park JW, Lee KH, et al. (2006) Repetitive transcrania magnetic stimulation-induced corticomotor excitability and associated motor skill acquisition in chronic stroke. Stroke 37: 1471-1476.

56. Ameli M, Grefkes C, Kemper F, Riegg FP, Rehme AK, et al. (2009) Differential effects of high-frequency repetitive transcranial magnetic stimulation over ipsilesional primary motor cortex in cortical and subcortical middle cerebral artery stroke. Ann Neurol 66: 298-309.

57. Hesse S, Werner C, Schonhardt EM, Bardeleben A, Jenrich W, et al. (2007) Combined transcranial direct current stimulation and robot-assisted arm training in subacute stroke patients: a pilot study. Restor Neurol Neurosci 25: 9-15.

58. Hsu WY, Cheng CH, Liao KK, Lee IH, Lin YY (2012) Effects of repetitive transcranial magnetic stimulation on motor functions in patients with stroke: a meta-analysis. Stroke 43: 1849-1857.

59. Rossi C, Sallustio F, Di Legge S, Stanzione P, Koch G (2013) Transcranial direct current stimulation of the affected hemisphere does not accelerate recovery of acute stroke patients. Eur J Neurol 20: 202-204.

60. Chang WH, Kim YH, Bang OY, Kim ST, Park YH, et al. (2010) Long-term effects of rTMS on motor recovery in patients after subacute stroke. J Rehabil Med 42: 758-764.

61. Emara TH, Moustafa RR, Elnahas NM, Elganzoury AM, Abdo TA, et al (2010) Repetitive transcranial magnetic stimulation at $1 \mathrm{~Hz}$ and $5 \mathrm{~Hz}$ produces sustained improvement in motor function and disability after ischaemic stroke. Eur J Neurol 17: 1203-1209.

62. Edwards DJ, Krebs HI, Rykman A, Zipse J, Thickbroom GW, et al. (2009) Raised corticomotor excitability of M1 forearm area following anodal tDCS is sustained during robotic wrist therapy in chronic stroke. Restor Neurol Neurosci 27: 199-207.

63. Malcolm MP, Triggs WJ, Light KE, Gonzalez Rothi LJ, Wu S, et al. (2007) Repetitive transcranial magnetic stimulation as an adjunct to constraintinduced therapy: an exploratory randomized controlled trial. Am J Phys Med Rehabil 86: 707-715.

64. Chang WH, Kim YH, Yoo WK, Goo KH, Park CH, et al. (2012) rTMS with moto training modulates cortico-basal ganglia-thalamocortical circuits in stroke patients. Restor Neurol Neurosci 30: 179-189.

65. Vines BW, Cerruti C, Schlaug G (2008) Dual-hemisphere tDCS facilitates greater improvements for healthy subjects' non-dominant hand compared to uni-hemisphere stimulation. BMC Neurosci 9: 103

66. Mordillo-Mateos L, Turpin-Fenoll L, Millán-Pascual J, Núñez-Pérez N Panyavin I, et al. (2012) Effects of simultaneous bilateral tDCS of the human motor cortex. Brain Stimul 5: 214-222.

67. Lindenberg R, Renga V, Zhu LL, Nair D, Schlaug G (2010) Bihemispheric brain stimulation facilitates motor recovery in chronic stroke patients. Neurology 75 : 2176-2184.

68. Lindenberg R, Zhu LL, Schlaug G (2012) Combined central and peripheral stimulation to facilitate motor recovery after stroke: the effect of number of sessions on outcome. Neurorehabil Neural Repair 26: 479-483.

69. Fusco A, De Angelis D, Morone G, Maglione L, Paolucci T, et al. (2013) The ABC of tDCS: Effects of Anodal, Bilateral and Cathodal Montages of Transcranial Direct Current Stimulation in Patients with Stroke-A Pilot Study. Stroke Res Treat 2013: 837595.

70. O'Shea J, Boudrias MH, Stagg CJ, Bachtiar V, Kischka U, et al. (2013) Predicting behavioural response to TDCS in chronic motor stroke. Neuroimage

71. Talelli P, Wallace A, Dileone M, Hoad D, Cheeran B, et al. (2012) Theta burst stimulation in the rehabilitation of the upper limb: a semirandomized, placebocontrolled trial in chronic stroke patients. Neurorehabil Neural Repair 26: 976987.

72. Hesse S, Waldner A, Mehrholz J, Tomelleri C, Pohl M,et al. (2011) Combined transcranial direct current stimulation and robot-assisted arm training in subacute stroke patients: an exploratory, randomized multicenter trial. Neurorehabil Neural Repair 25: 838-846.

73. Stagg CJ, Best JG, Stephenson MC, O'Shea J, Wylezinska M, et al. (2009)
Polarity-sensitive modulation of cortical neurotransmitters by transcranial stimulation. J Neurosci 29: 5202-5206.

74. Stagg CJ, Bachtiar V, Johansen-Berg $H$ (2011) The role of GABA in human motor learning. Curr Biol 21: 480-484.

75. Hamada M, Murase N, Hasan A, Balaratnam M, Rothwell JC (2013) The role of interneuron networks in driving human motor cortical plasticity. Cereb Cortex 23: $1593-1605$

76. Duncan PW, Goldstein LB, Horner RD, Landsman PB, Samsa GP, et al. (1994) Similar motor recovery of upper and lower extremities after stroke. Stroke 25 1181-1188.

77. Miyai I, Yagura H, Oda I, Konishi I, Eda H, et al. (2002) Premotor cortex is involved in restoration of gait in stroke. Ann Neurol 52: 188-194.

78. Miyai I, Yagura H, Hatakenaka M, Oda I, Konishi I, et al. (2003) Longitudinal optical imaging study for locomotor recovery after stroke. Stroke 34: 2866-2870.

79. Jayaram G, Stinear JW (2009) The effects of transcranial stimulation on paretic lower limb motor excitability during walking. J Clin Neurophysiol 26: 272-279.

80. Wang RY, Tseng HY, Liao KK, Wang CJ, Lai KL, et al. (2012) rTMS combined with task-oriented training to improve symmetry of interhemispheric corticomotor excitability and gait performance after stroke: a randomized trial. Neurorehabil Neural Repair 26: 222-230.

81. Madhavan S, Weber KA 2nd, Stinear JW (2011) Non-invasive brain stimulation enhances fine motor control of the hemiparetic ankle: implications for rehabilitation. Exp Brain Res 209: 9-17.

82. Kakuda W, Abo M, Watanabe S, Momosaki R, Hashimoto G, et al. (2013) Highfrequency rTMS applied over bilateral leg motor areas combined with mobility training for gait disturbance after stroke: a preliminary study. Brain Inj 27: 1080 1086 .

83. Kakuda W, Abo M, Nakayama Y, Kiyama A, Yoshida H (2013) High-frequency rTMS using a double cone coil for gait disturbance. Acta Neurol Scand 128 100-106.

84. Aglioti S, Dall'Agnola R, Girelli M, Marzi CA (1991) Bilateral hemispheric contro of foot distal movements: evidence from normal subjects. Cortex 27: 571-581.

85. Kapreli E, Athanasopoulos S, Papathanasiou M, Van Hecke P, Strimpakos N et al. (2006) Lateralization of brain activity during lower limb joints movement An fMRI study. Neuroimage 32: 1709-1721.

86. Roth $Y$, Zangen A, Hallett M (2002) A coil design for transcranial magnetic stimulation of deep brain regions. J Clin Neurophysiol 19: 361-370.

87. Zangen A, Roth Y, Voller B, Hallett M (2005) Transcranial magnetic stimulation of deep brain regions: evidence for efficacy of the $\mathrm{H}$-coil. Clin Neurophysio 116: $775-779$.

88. Levkovitz Y, Roth Y, Harel EV, Braw Y, Sheer A, et al. (2007) A randomized controlled feasibility and safety study of deep transcranial magnetic stimulation. Clin Neurophysiol 118: 2730-2744.

89. Levkovitz Y, Harel EV, Roth Y, Braw Y, Most D, et al. (2009) Deep transcrania magnetic stimulation over the prefrontal cortex: evaluation of antidepressant and cognitive effects in depressive patients. Brain Stimul 2: 188-200.

90. Harel EV, Zangen A, Roth Y, Reti I, Braw Y, et al. (2011) H-coil repetitive transcranial magnetic stimulation for the treatment of bipolar depression: an add-on, safety and feasibility study. World J Biol Psychiatry 12: 119-126.

91. Spagnolo F, Coppi E, Della Rosa PA, Fichera M, Barbieri A, et al. (2013) Deep magnetic stimulation in a progressive supranuclear palsy patient with speech involvement. J Neurol 260: 670-673.

92. Onesti E, Gabriele M, Cambieri C, Ceccanti M, Raccah R, et al. (2013) H-coil repetitive transcranial magnetic stimulation for pain relief in patients with diabetic neuropathy. Eur J Pain 17: 1347-1356.

93. Datta A, Bansal V, Diaz J, Patel J, Reato D, et al. (2009) Gyri-precise head model of transcranial direct current stimulation: improved spatial focality using a ring electrode versus conventional rectangular pad. Brain Stimul 2: 201-207, 207 e201.

94. Dmochowski JP, Datta A, Bikson M, Su Y, Parra LC (2011) Optimized multielectrode stimulation increases focality and intensity at target. J Neural Eng 8 : 046011. 